\title{
TRANSFER AND THE SPECTRAL SEQUENCE OF A FIBRATION
}

BY

\author{
CARLOS PRIETO
}

\begin{abstract}
ABSTRACr. The purpose of this paper is to show that transfers for fibrations behave well with respect to spectral sequences which are induced by filtrations of the base space. In particular, for the spectral sequence of a fibration (induced by the skeletal filtration of the base space), one obtains the expected effect on the $E_{2}$-terms: We prove that the transfer in the $E_{2}$-terms is determined by the transfer of the fiber (considered trivially as a fibration over a point). As an application, results of Atiyah on the $K$-theory of classifying spaces are transcribed to generalized cohomology theories.
\end{abstract}

This paper is a condensed version of Chapters I-III of my doctoral thesis [10], albeit without the assumption that the fibrations involved have to be orientable. I am indebted to Professor Albrecht Dold under whose direction I wrote my thesis.

Introduction. Let $\mathcal{T}$ be a class of commutative triangles of topological spaces and continuous maps

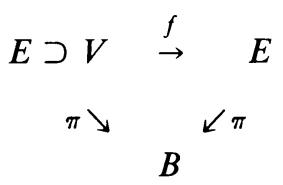

where $V$ is open in $E$. This class is to be closed under pullbacks, namely, if (0.1) lies in $\mathcal{T}$ and $g: B^{\prime} \rightarrow B$ is continuous, then the induced triangle

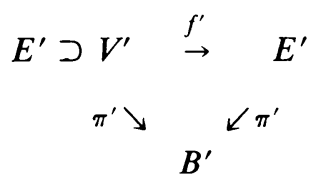

obtained by taking pullbacks over $g$ must lie in $\mathcal{T}$. We call such a class $\mathcal{T}$ a $\tau$-class.

Let $h$ be a cohomology (homology) theory and $\mathcal{T}$ a $\tau$-class; a transfer on $\mathcal{T}$ for $h$ will associate to each triangle (0.1) in $\mathcal{T}$ a homomorphism

$$
\tau(f): h\left(E, E_{A}\right) \rightarrow h(B, A)
$$

(or on the opposite direction if $h$ is a homology theory), called the transfer of (0.1), where $A \subset B$ and $E_{A}=\tau^{-1}(A)$.

Received by the editors November 26, 1980; presented to the AMS at the 775th meeting, April 12, 1980, Indiana University, Bloomington.

1980 Mathematics Subject Classification. Primary 55N20, 55R05, 55R20; Secondary 55T10, 55T25, 55M20, 55R 35.

Key words and phrases. Transfer, spectral sequence of a filtration, spectral sequence of a fibration, Atiyah-Hirzebruch-Whitehead spectral sequence, Leray-Serre spectral sequence. 
Such a transfer is to have the following properties: (we shall formulate only the cohomology case; for homology one has corresponding properties)

0.3. Naturality. If $g: B^{\prime} \rightarrow B$ is continuous and $A^{\prime} \subset B^{\prime}$ is such that $g\left(A^{\prime}\right) \subset A$, then the diagram

$$
\begin{array}{lrr}
h\left(E, E_{A}\right) & \stackrel{\bar{g}^{*}}{\rightarrow} & h\left(E^{\prime}, E_{A^{\prime}}^{\prime}\right) \\
\tau(f) \downarrow & & \downarrow \tau\left(f^{\prime}\right) \\
h(B, A) & \stackrel{g^{*}}{\rightarrow} & h\left(B^{\prime}, A^{\prime}\right)
\end{array}
$$

is commutative, where $\tau\left(f^{\prime}\right)$ is the transfer of $(0.2)$ and $\bar{g}: E^{\prime} \rightarrow E$ is the canonical map.

0.5. Stability. Let $\left(\Delta^{p}, \dot{\Delta}^{p}\right)$ be the elementary $p$-simplex and its boundary. Pulling back (0.1) over the projection $\Delta^{p} \times B \rightarrow B$ we get the triangle

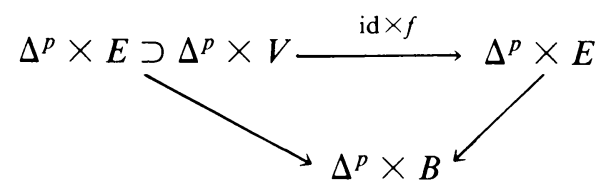

which admits a transfer such that the square

$$
\begin{array}{ccc}
h^{q}\left(E, E_{A}\right) & \stackrel{\sigma^{p}}{\rightarrow} & h^{p+q}\left(\left(\Delta^{p}, \dot{\Delta}^{p}\right) \times\left(E, E_{A}\right)\right) \\
\tau(f) \downarrow & & \downarrow \tau(\mathrm{id} \times f) \\
h^{q}(B, A) & \stackrel{\sigma^{p}}{\rightarrow} & h^{p+q}\left(\left(\Delta^{p}, \dot{\Delta}^{p}\right) \times(B, A)\right)
\end{array}
$$

commutes, where $\sigma^{p}$ denotes the $p$-iterated suspension isomorphism.

0.8 . Equivalent to 0.5 is the compatibility with the boundary homomorphisms of the cohomology theory, i.e. $\tau(f) \delta=\delta \tau(f)$.

Besides these general properties of the transfer, one has another which will be of special interest in what follows.

0.9 . If the triangle

$$
\begin{array}{cccc}
F \supset U & \stackrel{f_{0}}{\rightarrow} & F \\
\pi_{0} \searrow & & \swarrow \pi_{0}
\end{array}
$$

lies in a $\tau$-class admitting a transfer for a theory $h$ and for singular cohomology $H$, then

$$
\tau\left(f_{0}\right) \pi_{0}^{*}=\Lambda\left(f_{0}\right) \cdot: h(*) \rightarrow h(*)
$$

where $\Lambda\left(f_{0}\right)=\tau(f)(1) \in H^{0}(*)=\mathbf{Z}, \quad l \in H^{0}(F)$ for the transfer in singular cohomology. This number is called the Lefschetz-Hopf index of $f_{0}$.

0.10 . N.B. In many cases $\Lambda\left(f_{0}\right)$ agrees with the Lefschetz-number of $f_{0}$ (when it is defined), see $[3, \S \S 2,3]$ and/or [6, VII.6.6]. 
0.11. As a corollary of 0.9 it follows that if $F$ is 0 -connected, then $\tau\left(f_{0}\right)=\Lambda\left(f_{0}\right) \eta^{*}$ : $h(F) \rightarrow h(*)$, where $\eta: * \rightarrow F$ is any map.

Up to here we have talked quite abstractly about the transfer. I shall now mention some concrete examples.

0.12 . If $\mathcal{T}$ is the class of all finite coverings $\pi: E \rightarrow B$ (considered as triangles with $V=E$ and $f=$ id) one has the Atiyah-transfer [2] (direct image) for $K$-theory

$$
\tau(\text { id })=\pi_{!}: K(E) \rightarrow K(B)
$$

and the transfer first defined by Eckmann [8] in singular theories, say

$$
\tau(\text { id }): H^{*}(E) \rightarrow H^{*}(B) \text {. }
$$

This was extended to all generalized cohomology theories, since it is induced by a suitable map of spectra as shown by Roush. See [1] for a detailed discussion.

0.13 . For the class $\mathscr{T}$ of triangles (0.1) such that $\pi: E \rightarrow B$ is an $E N R_{B}$ with $B$ metric and $f$ compactly fixed (and all their pullbacks) Dold constructed a transfer in [7] for all cohomology theories (over $B$ ), and

0.14 . For the class $\mathcal{T}$ of triangles $(0.1)$ such that $\pi: E \rightarrow B$ is a Hurewicz-fibration with fiber $F$ (homotopy-equivalent to) a finite $\mathrm{CW}$-complex, basis $B$ (homotopyequivalent to) a finite dimensional $\mathrm{CW}$-complex and $V=E$ (and the pullbacks of such triangles), Becker and Gottlieb [3] defined also a transfer, further generalized by M. Clapp de Prieto $[4,5]$ to a basis $B$ without any restriction on the dimension.

In all cases one may show that the constructed transfers have all mentioned properties (and others).

1. The transfer and the spectral sequence of a filtration. From now on we shall say briefly that a given triangle (0.1) admits a transfer for a theory $h$ if it belongs to a $\tau$-class $\mathcal{T}$ for which a transfer for $h$ can be defined.

1.1. Let (0.1) admit a transfer and take a filtration of the pair $(B, A)$

$$
B: A=B^{-1} \subset B^{0} \subset \cdots \subset B^{n} \subset B^{n+1} \subset \cdots \subset B^{\infty}=B
$$

and the induced filtration of the pair $\left(E, E_{A}\right)$

$$
\text { E: } E_{A}=E^{-1} \subset E^{0} \subset \cdots \subset E^{n} \subset E^{n+1} \subset \cdots \subset E^{\infty}=E,
$$

that is, $E^{n}=\pi^{-1}\left(B^{n}\right), n=-1,0,1, \ldots, \infty$. Take $k \leqslant l \leqslant m \leqslant n$ and consider

$$
\begin{array}{lllllll}
E^{k} & \hookrightarrow & E^{l} & \hookrightarrow & E^{m} & \hookrightarrow & E^{n} \\
\downarrow & & \downarrow & & \downarrow & & \downarrow \\
B^{k} & \hookrightarrow & B^{l} & \hookrightarrow & B^{m} & \hookrightarrow & B^{n}
\end{array}
$$

where all squares are pullback diagrams. From the naturality of the transfer, 0.3 , we know that the square

$$
\begin{array}{ccc}
h\left(E^{n}, E^{l}\right) & \rightarrow & h\left(E^{n}, E^{k}\right) \\
\tau \downarrow & & \downarrow \tau \\
h\left(B^{n}, B^{l}\right) & \rightarrow & h\left(B^{n}, B^{k}\right)
\end{array}
$$

commutes, where the horizontal arrows are induced by the corresponding inclusions and $\tau$ denotes the transfers of the corresponding restrictions of $f$. 
The compatibility of $\tau$ with boundary homomorphisms 0.8 gives us the commutative square

$$
\begin{array}{ccc}
h\left(E^{l}, E^{k}\right) & \stackrel{\delta}{\rightarrow} & h\left(E^{m}, E^{l}\right) \\
\tau \downarrow & & \downarrow \tau \\
h\left(B^{l}, B^{k}\right) & \stackrel{\delta}{\rightarrow} & h\left(B^{m}, B^{l}\right)
\end{array}
$$

where $\delta$ denotes the boundary homomorphisms of the triples $E^{k} \subset E^{l} \subset E^{m}$ and $B^{k} \subset B^{l} \subset B^{m}$ respectively.

Since all groups mentioned and all horizontal arrows are the ones taken into account for defining the spectral sequences of the filtrations $\mathscr{B}$ and $\mathcal{E}$ respectively, it is straightforward to prove using (1.2) and (1.3) the following

1.4. THEOREM. $\tau(f)$ induces a transformation of spectral sequences

$$
\tau:\left\{E_{r}, d_{r}\right\} \rightarrow\left\{\bar{E}_{r}, \bar{d}_{r}\right\}
$$

where $\left\{E_{r}, d_{r}\right\}$ is the spectral sequence induced by the filtration $\mathcal{E}$ and $\left\{\bar{E}_{r}, \bar{d}_{r}\right\}$ the one induced by $\mathscr{B}$.

(Cf. $[11,15.56]$ for the definition of a transformation of spectral sequences.)

\section{The transfer and the spectral sequence of a fibration.}

2.1. To begin, let us recall the theorem of Leray-Serre for general theories.

Suppose $\pi: E \rightarrow B$ is a (Hurewicz-) fibration and $A \subset B$ is so that $(B, A)$ is a 0 -connected relative $\mathrm{CW}$-complex., Take as $\mathscr{B}$ in $\S 1$ the skeletal filtration of $(B, A)$ and as $\mathcal{E}$ the induced filtration of $\left(E, E_{A}\right)$. Let $h$ be a strongly additive cohomology theory (or just an arbitrary one if $(B, A)$ has only finitely many cells in each dimension). One has

2.2. TheOREm (LeRAY-Serre). The spectral sequence associated to $\mathcal{E},\left\{E_{r}, d_{r}\right\}$, converges to $h\left(E, E_{A}\right)$ and there is an isomorphism

$$
E_{2}^{p q} \cong H^{p}\left(B, A ; h^{q}(\mathscr{F})\right)
$$

where $H^{*}$ means singular (or cellular) cohomology with coefficients taken in the local system of coefficients given by $h^{q}\left(\pi^{-1}(b)\right), b \in B$, as usual (and correspondingly for $a$ homology theory).

I shall not prove the theorem and just refer the reader to [12, XII.4.9 and 4.9*]. I will only show what the isomorphism looks like.

2.3. First of all I describe the local system $h(\mathscr{F})$. This is a contravariant functor

$$
h(\mathscr{F}): \Pi_{1}(B) \rightarrow \mathrm{Ab}
$$

from the fundamental groupoid of $B$ to abelian groups, such that

$$
h(\mathscr{F})(b)=h\left(F_{b}\right), \quad F_{b}=\pi^{-1}(b)
$$

and if $\omega: I \rightarrow B$ is a path from $b_{0}$ to $b_{1}$ then $h(\mathscr{F})(\omega)=\bar{\omega}^{*}$, where $\bar{\omega}: F_{b_{0}} \rightarrow F_{b_{1}}$ is given by $\bar{\omega}(y)=\lambda(y, \omega)(1)$ and $\lambda(y, \omega)$ is a path over $\omega$ beginning at $y$ and lying over $\omega$ (say, given by a lifting function $\lambda$ ). 
2.4. The cellular cocomplex with coefficients in $h^{q}(\mathscr{F})$ associated to the skeletal filtration $\Re$ of $(B, A)$ has as $p$ th group

$$
\Gamma^{p}\left(B, A ; h^{q}(\mathscr{F})\right)=\prod_{\phi \in \Phi_{p}} h^{q}\left(F_{\phi\left(e_{0}\right)}\right)
$$

where $\Phi_{p}$ is the set of all characteristic maps of the $p$-cells of $(B, A)$ considered as maps $\phi:\left(\Delta^{p}, \dot{\Delta}^{p}\right) \rightarrow\left(B^{p}, B^{p-1}\right)$ and $e_{0}=(1,0,0, \ldots, 0) \in \Delta^{p} \subset \mathbf{R}^{p+1}$ (cf. [12]).

2.5. Take a characteristic map $\phi$ as before and pull back $E^{p} \rightarrow B^{p}$ over $\phi$ :

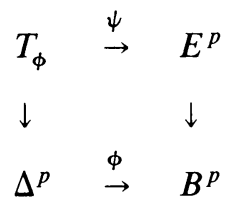

Since $\Delta^{p}$ is contractible (to $e_{0}$ ), there is a (well-defined, up to vertical homotopy) trivialization

$$
\alpha_{\phi}: \Delta^{p} \times F_{\phi\left(e_{0}\right)} \stackrel{\simeq}{\rightarrow} T_{\phi}
$$

(i.e. fiber homotopy equivalence such that $\alpha_{\phi}\left(e_{0},-\right) \simeq$ id). The isomorphism $\kappa$ : $E_{1}^{p q}=h^{p+q}\left(E^{p}, E^{p-1}\right) \rightarrow \Gamma^{p}\left(B, A ; h^{q}(\mathscr{F})\right)$ is given by the composite

$$
h^{p+q}\left(E^{p}, E^{p-1}\right) \stackrel{\psi^{*}}{\rightarrow} h^{p+q}\left(T_{\phi}, \dot{T}_{\phi}\right) \stackrel{\alpha_{\phi}^{*}}{\cong} h^{p+q}\left(\left(\Delta^{p}, \dot{\Delta}^{p}\right) \times F_{\phi\left(e_{0}\right)}\right) \stackrel{\sigma^{p}}{\cong} h^{q}\left(F_{\phi\left(e_{0}\right)}\right)
$$

in each factor, where $\dot{T}_{\phi}$ is the restriction of $T_{\phi}$ to the boundary of $\Delta^{p}, \dot{\Delta}^{p}$, and $\sigma^{p}$ is the $p$-iterated suspension isomorphism.

2.6. In case that $E=B$ and the projection is the identity, then the local coefficient system $h^{q}(\mathscr{F})$ becomes the global coefficient group $h^{q}(*)$ and $\kappa$ turns out to be very simple; that is, $\bar{\kappa}: \bar{E}_{1}^{p q}=h^{p+q}\left(B^{p}, B^{p-1}\right) \rightarrow \Gamma^{p}\left(B, A ; h^{q}(*)\right)$ is defined by

$$
h^{p+q}\left(B^{p}, B^{p-1}\right) \stackrel{\phi^{*}}{\rightarrow} h^{p+q}\left(\Delta^{p}, \dot{\Delta}^{p}\right) \stackrel{\sigma^{p}}{\longleftarrow} h^{q}(*) .
$$

This $\bar{\kappa}$ gives the isomorphism of the Atiyah-Hirzebruch-Whitehead spectral sequence.

2.7. Let now $\tilde{\pi}: \tilde{E} \rightarrow B \times I$ be a Hurewicz-fibration with lifting map $\tilde{\lambda}$. Let $i_{\nu}$ : $B \approx B \times\{\nu\} \hookrightarrow B \times I$ be the inclusion $(\nu=0,1)$. Call $E^{\nu} \subset \tilde{E}$ the restriction (pullback) of $E$ over $i_{v}$. We shall construct a homotopy equivalence over $B, \bar{\omega}: E^{0} \rightarrow E^{1}$ as follows. Given $e \in \tilde{E}$ let $\tilde{\pi} e=(\pi e, s e) \in B \times I$, whence $e \in E^{\nu}$ if and only if $s e=\nu$. For $e \in E^{0}$ define the path $\omega_{e}: I \rightarrow B \times I$ by $\omega_{e}(t)=(\pi e, t)$. Now let

$$
\bar{\omega}(e)=\tilde{\lambda}\left(e, \omega_{e}\right)(1) \in E^{1}
$$

which is well defined since $\tilde{\pi} e=(\pi e, 0)=\omega_{e}(0)$. One may easily show that this is a fiber homotopy equivalence.

The following lemma will be of interest in what follows.

2.8. Lemma. Suppose that the triangle

$$
\begin{array}{rlrl}
\tilde{E} \supset \tilde{V} & \stackrel{\tilde{f}}{\rightarrow} \quad \tilde{E} \\
\tilde{\pi} \searrow & & \multicolumn{2}{r}{} \\
& B \times I
\end{array}
$$


admits a transfer for $h$, where $\tilde{\pi}$ is as above, and let $A \subset B$. If $f^{\nu}: V^{\nu} \rightarrow E^{\nu}$ denotes the restriction of $\tilde{f}$ to $B \times\{\nu\}$, then the diagram

$$
\begin{array}{rll}
h\left(E^{0}, E_{A}^{0}\right) & \stackrel{\bar{\omega}^{*}}{\leftrightarrows} & h\left(E^{1}, E_{A}^{1}\right) \\
\tau\left(f^{0}\right) \searrow & & \swarrow \tau\left(f^{1}\right) \\
& h(B, A) &
\end{array}
$$

is commutative.

Proof. Let $j_{\nu}: E^{\nu} \rightarrow \tilde{E}$ be the inclusion. By naturality we obtain

$$
\begin{array}{ccccr}
h\left(E^{0}, E_{A}^{0}\right) & \stackrel{j_{0}^{*}}{\leftarrow} & h\left(\tilde{E}, \tilde{E}_{A \times I}\right) & \stackrel{j^{*}}{\rightarrow} & h\left(E^{1}, E_{A}^{1}\right) \\
\downarrow \tau\left(f^{0}\right) & & \downarrow \tau(\tilde{f}) & & \downarrow \tau\left(f^{1}\right) \\
h(B, A) & \stackrel{i_{0}^{*}}{\leftrightarrows} & h((B, A) \times I) & \stackrel{i_{1}^{*}}{\leftrightarrows} & h(B, A) .
\end{array}
$$

Whence $\tau\left(f^{0}\right) j_{0}^{*}=\tau\left(f^{1}\right) j_{1}^{*}$ since $i_{1}^{*}\left(i_{0}^{*}\right)^{-1}=1$. We now construct a map $k: \tilde{E} \rightarrow E^{1}$ such that (i) $k j_{1} \simeq \mathrm{id}_{E^{1}}$ and (ii) $k j_{0}=\bar{\omega}$, thus proving the lemma: For $e \in E$ let $\tilde{\omega}_{e}(t)=(\pi e,(1-s e) t+s e)$. One may easily check that

$$
k(e)=\tilde{\lambda}\left(e, \tilde{\omega}_{e}\right)(1)
$$

is well defined and satisfies (i) and (ii).

We apply this lemma to obtain the following

2.9. Proposition. Let (0.1) admit a transfer and be such that $\pi$ is a fibration. Then the transfer defines a transformation of coefficient systems

$$
\tau: h(\mathscr{F}) \rightarrow h(*)
$$

such that for each $b \in B, \tau_{b}=\tau\left(f_{b}\right): h\left(F_{b}\right) \rightarrow h(*)$ for the pullback diagram

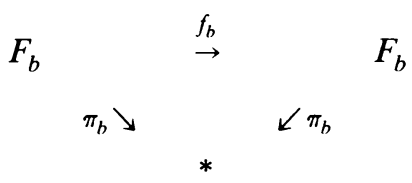

over $* \rightarrow\{b\} \hookrightarrow B$.

Proof. Let $\omega: I \rightarrow B$ be a path from $b_{0}$ to $b_{1}$. If $\tilde{\pi}: \tilde{E} \rightarrow I$ is the pullback of $E$ over $\omega$ we fall in the case described above and the $\bar{\omega}: F_{b_{0}} \rightarrow F_{b_{1}}$ defined in 2.3 agrees with the $\bar{\omega}: E^{0} \rightarrow E^{1}$ defined in 2.7. Hence the lemma states that the diagram

$$
\begin{array}{lcc}
h\left(F_{b_{0}}\right) \stackrel{\tau\left(f_{b_{0}}\right)}{\rightarrow} & h(*) \\
\bar{\omega}^{*} \downarrow & & \| \\
h\left(F_{b_{1}}\right) & \stackrel{\tau\left(f_{b_{b}}\right)}{\rightarrow} & h(*)
\end{array}
$$

commutes, thus proving the naturality of $\tau$.

We need also the following corollary of 2.8 . 
2.10. Proposition. In the situation (0.1) let $\pi: E \rightarrow B$ be a fibration with $B$ contractible and take a trivialization $\alpha: B \times F \rightarrow E$. Then the triangle

$$
\begin{array}{clc}
h\left(E, E_{A}\right) & \stackrel{\alpha^{*}}{\rightarrow} \quad h((B, A) \times F) \\
\tau(f) \searrow & & \swarrow \tau\left(\mathrm{id} \times f_{0}\right) \\
& h(B, A)
\end{array}
$$

commutes, where $f_{0}$ is the restriction of $f$ to the point $b_{0} \in B$ to which $B$ contracts.

Proof. Let $H: B \times I \rightarrow B$ be a contraction such that $H(b, 0)=b_{0}, H(b, 1)=b$. Take the induced triangle

$$
\begin{aligned}
\tilde{E} \supset \tilde{V} & \stackrel{\tilde{f}}{\rightarrow} \tilde{E} \\
\tilde{\pi} \searrow & \\
& B \times I
\end{aligned}
$$

by pulling back $(0.1)$ over $H$. From Lemma 2.8 we know that

$$
\tau\left(f^{0}\right) \bar{\omega}^{*}=\tau\left(f^{1}\right)
$$

holds. We have that $E^{0}$ is the pullback of $E$ over the map $B \rightarrow\left\{b_{0}\right\} \hookrightarrow B$, whence $E^{0}=B \times F\left(F=\pi^{-1}\left(b_{0}\right)\right)$ and $f^{0}: B \times(F \cap V) \rightarrow B \times F$ is such that $f^{0}(b, e)=$ $(b, f(e))$, i.e. $f^{0}=\mathrm{id} \times f_{0} .\left(f_{0}=f \mid F\right.$.) On the other hand $E^{1}$ is the pullback of $E$ over the identity, thus $E^{1}=E$ and $f^{1}=f$. Under these identifications $\bar{\omega}$ becomes a trivialization which is vertically homotopic to $\alpha$, whence (1) becomes

$$
\tau\left(\mathrm{id} \times f_{0}\right) \alpha^{*}=\tau(f)
$$

as desired.

2.11. THEOREM. The diagram

$$
\begin{array}{crr}
h^{p+q}\left(E^{p}, E^{p-1}\right) & \stackrel{\kappa}{\rightarrow} & \Gamma^{p}\left(B, A ; h^{q}(\mathcal{F})\right) \\
\tau(f) \downarrow & & \downarrow \Gamma^{p}(B, A ; \tau) \\
h^{p+q}\left(B^{p}, B^{p-1}\right) & \stackrel{\kappa}{\rightarrow} & \Gamma^{p}\left(B, A ; h^{q}(*)\right)
\end{array}
$$

is commutative.

Proof. It is enough to check the commutativity of the diagram

$$
\begin{aligned}
& h^{p+q}\left(E^{p}, E^{p-1}\right) \stackrel{\psi^{*}}{\rightarrow} h^{p+q}\left(T_{\phi}, \dot{T}_{\phi}\right) \stackrel{\alpha_{\phi}^{*}}{\rightarrow} h^{p+q}\left(\left(\Delta^{p}, \dot{\Delta}^{p}\right) \times F_{\phi\left(e_{0}\right)}\right) \stackrel{\sigma^{-p}}{\rightarrow} h^{q}\left(F_{\phi\left(e_{0}\right)}\right)
\end{aligned}
$$

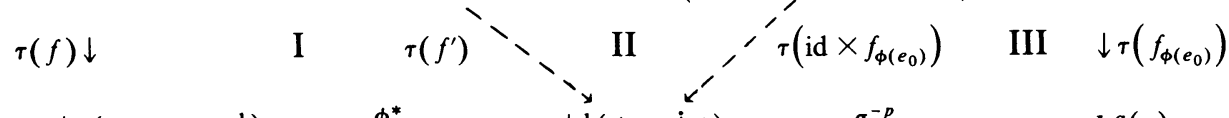

$$
\begin{aligned}
& h^{p+q}\left(B^{p}, B^{p-1}\right) \longrightarrow \phi^{*} \longrightarrow h^{p+1}\left(\Delta^{p}, \dot{\Delta}^{p}\right) \longrightarrow \sigma^{q}(*)
\end{aligned}
$$

which can be performed by doing it separately for the subdiagrams I, II and III. I commutes by the naturality, 0.3 , of $\tau(f)$, III commutes by the stability, 0.5 , of $\tau\left(f_{\phi\left(e_{0}\right)}\right)$. The commutativity of II follows from 2.10 . 
We obtain our main theorem now immediately as a corollary of 2.11 :

2.12. Theorem. Consider the triangle (0.1) such that $\pi: E \rightarrow B$ is a fibration over a relative $\mathrm{CW}$-complex $(B, A)$, and a strongly additive cohomology theory $h$ for which it admits a transfer. Then this transfer induces a transformation from the spectral sequence of the fibration to the Atiyah-Hirzebruch-Whitehead spectral sequence of $B$

$$
\tau(f):\left\{E_{r}, d_{r}\right\} \rightarrow\left\{\bar{E}_{r}, \bar{d}_{r}\right\}
$$

converging to $\tau(f): h\left(E, E_{A}\right) \rightarrow h(B, A)$ in such a way that for the $E_{2}$-terms, the diagram

$$
\begin{aligned}
& E_{2}^{p q} \cong H^{p}\left(B, A ; h^{q}(\mathscr{F})\right) \\
& \tau(f) \downarrow \quad \downarrow H^{p}(B, A ; \tau) \\
& \bar{E}_{2}^{p q} \cong H^{p}\left(B, A ; h^{q}(*)\right)
\end{aligned}
$$

commutes, where $\tau_{b}=\tau\left(f_{b}\right): h^{q}\left(F_{b}\right) \rightarrow h^{q}(*)$ is the transfer of the restriction to each fiber.

N.B. For a cohomology theory which sends weak homotopy equivalences to isomorphisms, there is, of course, no need to restrict ourselves to relative $\mathrm{CW}$ complexes: the theorem holds for any pair $(B, A)$ by giving a $C W$-approximation of it as usual.

2.13. There is a homology version of all this, which can be obtained in the same way. I omit the details.

3. Applications. We apply 2.12 to vary some results in [2].

3.1. Suppose that $B$ is a $\mathrm{CW}$-complex and recall that

$$
\begin{aligned}
& F^{n}=\operatorname{Ker}\left(h(E) \rightarrow h\left(E^{n-1}\right)\right), \\
& \bar{F}^{n}=\operatorname{Ker}\left(h(B) \rightarrow h\left(B^{n-1}\right)\right),
\end{aligned}
$$

by definition. Define the associated graded groups of the filtered groups $h(E)$ and $h(B)$ by

$$
\operatorname{Sh}(E)=\underset{n}{\bigoplus} F^{n} / F^{n+1}=\underset{n}{\bigoplus} E_{\infty}^{n}
$$

and

$$
\operatorname{Sh}(B)=\underset{n}{\oplus} \bar{F}^{n} / \bar{F}^{n+1}=\underset{n}{\bigoplus} \bar{E}_{\infty}^{n} .
$$

3.2. Theorem (CF. $[2,2.11])$. Take a fibration $\pi: E \rightarrow B$ and a map $f: V^{\prime} \rightarrow E(V$ open in $E)$ over $B$ such that it has a transfer. Then $\Lambda=\Lambda\left(f_{0}\right)\left(f_{0}=f \mid F \cap V\right.$, $\left.F=\pi^{-1}\left(b_{0}\right)\right)$ annihilates the kernel of

$$
\pi^{*}: \operatorname{Sh}(B) \rightarrow \operatorname{Sh}(E) .
$$

If $p$ is a prime number not dividing $\Lambda, H^{n}(B)$ is finitely generated for all $n$ (e.g. if the $n$-skeleton $B^{n}$ is finite for all $\left.n\right)$ and $h(*)$ is a finitely generated group, then the p-primary component of $\operatorname{Sh}(B)$ is a direct summand of $\operatorname{Sh}(E)$. Moreover, if the filtiation of $h(B)$ is finite (e.g. if $B$ is finite), then the p-primary component of $h(B)$ is a direct summand of $h(E)$. 
Proof. The composite

$$
\tau(f) \pi^{*}: \operatorname{Sh}(B) \rightarrow \operatorname{Sh}(B)
$$

is multiplication by $\Lambda$, since this is the case in the $E_{2}$-terms and whence also in the $E_{\infty}$-terms. Thus the first part of the theorem.

Let now $T^{p}(n)$ be the $p$-primary component of $\bar{E}_{\infty}^{n}$ which is a direct summand by the theorem of primary decomposition, since $\bar{E}_{\infty}^{n}$ is finitely generated as one may prove by using the spectral sequence $\bar{E}_{r}^{n}$ and the assumptions on $H^{*}(B)$ and $h(*)$. The diagram

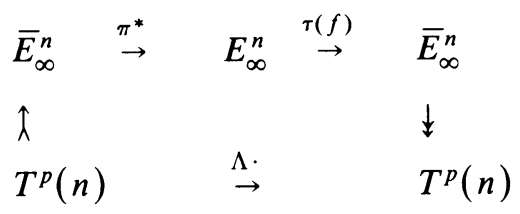

commutes; the lower arrow is mono, since $p$ does not divide $\Lambda$, in consequence it is also epi $\left(T^{p}(n)\right.$ is finite). This proves the second part. The third is now an easy consequence of the second part.

3.3. COROLlary (CF. $[2,4.8])$. Let $G$ be a finite group and BG its classifying space. Then the order of $G, o(G)$, annihilates $\operatorname{S} \tilde{h}(B G)$. $(\mathcal{G} \tilde{h}(B G)$ is constructed from the filtration $\tilde{F}^{n}=\operatorname{Ker}\left(\tilde{h}(B G) \rightarrow \tilde{h}\left(B^{n-1}\right)\right)$, with $B^{n-1}$ the $(n-1)$-skeleton of $B G ; F^{0}=$ $h(B G)$.)

Proof. Take the triangle

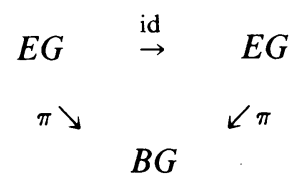

where $\pi: E G \rightarrow B G$ is the classifying bundle of $G$. It does admit a transfer, and since $h(E G)=h(*)$ it follows that $\operatorname{Ker}(\mathscr{S} h(B G) \rightarrow \mathcal{G}(E G))=\mathscr{S} \tilde{h}(B G)$. Apply the first part of 3.2.

3.4. COROLlaRY (CF. $[2,4.9])$. Suppose that $h(*)$ is a finitely generated group. If $G$ is a finite group, then the p-primary component of $\operatorname{Sh}(B G)$ is a direct summand of $\operatorname{Sh}(B H)$ for each p-Sylow subgroup $H$ of $G$.

Proof. The triangle

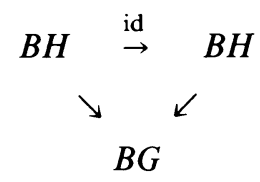

has $F=G / H$ as fiber, and under our hypotheses $\chi_{F}=[G: H]$ is not divisible by $p$; whence the result follows from the second part of 3.5 .

3.5. N.B. Under the same hypotheses of 3.4, it follows from the third part of 3.2 that the p-primary component of $h\left(B^{n} G\right)$ is a direct summand of $h\left(B^{n} H\right)$, where $B^{n} G$ and $B^{n} H$ are the corresponding $n$-skeleta. 


\section{REFERENCES}

1. J. F. Adams, Infinite loop spaces, Ann. of Math. Studies, no. 90, Princeton Univ. Press, Princeton, N.J., 1978.

2. M. F. Atiyah, Characters and cohomologv of finite groups, IHES Publ. Math. 9 (1961), 247-288.

3. J. C. Becker and D. H. Gottlieb, Transfer maps for fibrations and duality, Compositio Math. 33 (1976), 107-133.

4. M. Clapp de Prieto, Dualität in der Kategorie der Spektren von Ex-Räumen, Thesis, Heidelberg, 1979.

5. __ Duality and transfer for parametrized spectra, Arch. Math. (to appear).

6. A. Dold, Lectures in algebraic topology, Springer-Verlag, Berlin and New York, 1972.

7. __ The fixed-point transfer for fibre-preserving maps, Math. Z. 148 (1976), 215-244.

8. B. Eckmann, On complexes with operators, Proc. Nat. Acad. Sci. U.S.A. 39 (1953), 35-42.

9. J. W. Milnor, Construction of universal bundles. II, Ann. of Math. (2) 63 (1956), 430-436.

10. C. Prieto, Transfer und Spektralsequenzen, Thesis, Heidelberg, 1979.

11. R. M. Switzer, Algebraic topology-Homotopy and homology, Springer-Verlag, Berlin-Heidelberg-New York, 1975.

12. G. W. Whitehead, Elements of homotopy theory, Springer-Verlag, Berlin and New York, 1978.

Instituto de Matemáticas, U.N.A.M., 04360 Mexico, D. F., MExico 